\title{
$d$-wave resonating valence bond states of fermionic atoms in optical lattices
}

\author{
Simon Trebst ${ }^{1,2,3}$, Ulrich Schollwöck ${ }^{4}$, Matthias Troyer ${ }^{1}$, Peter Zoller ${ }^{5}$ \\ (1) Theoretische Physik, Eidgenössische Technische Hochschule Zürich, CH-8093 Zürich, Switzerland \\ ${ }^{(2)}$ Computational Laboratory, Eidgenössische Technische Hochschule Zürich, CH-8092 Zürich, Switzerland \\ (3) Microsoft Research and Kavli Institute for Theoretical Physics, \\ University of California, Santa Barbara, CA 93106, USA \\ (4) Institut für Theoretische Physik C, RWTH Aachen, D-52056 Aachen, Germany and \\ ${ }^{(5)}$ Institute for Theoretical Physics, University of Innsbruck, and \\ Institute for Quantum Optics and Quantum Information of the Austrian Academy of Science, 6020 Innsbruck, Austria
}

(Dated: December 3, 2018)

\begin{abstract}
We study controlled generation and measurement of superfluid $d$-wave resonating valence bond (RVB) states of fermionic atoms in $2 \mathrm{D}$ optical lattices. Starting from loading spatial and spin patterns of atoms in optical superlattices as pure quantum states from a Fermi gas, we adiabatically transform this state to an RVB state by change of the lattice parameters. Results of exact timedependent numerical studies for ladders systems are presented, suggesting generation of RVB states on timescale smaller than typical experimental decoherence times.
\end{abstract}

Resonating valence bond (RVB) states, in which electrons are paired into short-range singlets by a purely electronic mechanism, were originally introduced [1] as wave functions for insulating spin liquid ground states of Mott insulators. Shortly after the observation of high-temperature superconductivity in the cuprates [2], Anderson conjectured that they might be described by doped RVB states [3] , in which the RVB pairs of the insulating state become mobile and superconducting upon doping. Since the predicted exotic $d$-wave pairing symmetry [4] of the RVB pairs was confirmed experimentally in the cuprate superconductors [5], the RVB scenario remains as one of the most promising contenders for the theory of high temperature superconductivity [ 6 . While the ground state of the lightly doped two-dimensional Hubbard model is still unknown, the $d$-wave RVB scenario has been confirmed for $t$ - $J$ and Hubbard models of coupled plaquettes [7, [8] and ladders (consisting of two coupled chains) [9] by numerical simulations. The key question is whether the RVB state of weakly coupled ladders [10] or plaquettes 8] is connected to a possible RVB ground state of the square lattice Hubbard model: does the RVB state survive when the inter-plaquette (ladder) coupling is increased and becomes the same as the intraplaquette (ladder) coupling, at which point we have a uniform square lattice?

We propose to address this question using cold atoms loaded in optical lattices, which allow the realization of Hubbard models with controllable parameters, promising an entirely new avenue in the study of strongly correlated systems $11,12,13,14,15,16,17,18$. In particular, formation of RVB ground states with fermionic atoms in a $2 \mathrm{D}$ geometry is one of the ultimate challenges 12]. However, d-wave pair binding energies are two orders of magnitude smaller than the hopping amplitudes [8] for atoms in an optical lattice which are typically in the $\mathrm{kHz}$ range: this poses a significant experimental challenge in terms of temperature requirements etc., which will be difficult to meet. Instead we propose here the formation of RVB states by loading spatial and spin patterns of atoms in optical superlattices as pure quantum states from a reservoir of a quantum degenerate Fermi gas [19], and adiabatically transforming this state to an RVB state by change of the lattice parameters. An initial lattice configuration is designed in such a way that the desired initial state corresponds to the ground state of the deformed lattice in the form of a simple many-body product state, with a large excitation gap. This makes the preparation of these states robust against imperfections. In choosing a protocol for the deformation of the lattice to achieve the RVB state, the challenge is to select a parameter path, which minimizes the number of possible avoided crossings, and which sets the time scale for the adiabatic transformation, reminiscent of discussions in adiabatic quantum computing [20]. While this technique is illustrated for the case of RVB states here, it might provide a generic procedure to generate non-trivial ground states of dilute fermionic gases in lattices.

In this Letter we present a detailed investigation of this scenario. We will start with the loading of spin and spatial patterns on decoupled plaquettes formed by optical superlattices representing different atomic dopings, and discuss requirements for the formation of RVB plaquette states, and possible experimental signatures demonstrating $d$-wave pairing. We present results of exact timedependent numerical studies for ladders systems, suggesting that RVB states can be generated on a timescale smaller than the typical decoherence time of atoms in optical lattices. Since the numerical simulation of $2 \mathrm{D}$ strongly correlated systems belongs to the class of nondeterministic polynomial-time hard (NP-hard) computational problems [21], we are not able to provide answers for the coupling of plaquettes or ladders to probe the ground state of the 2D Hubbard model, but pose this as an important problem left for experiments to be solved.

Optical lattices - We start by summarizing the basic 


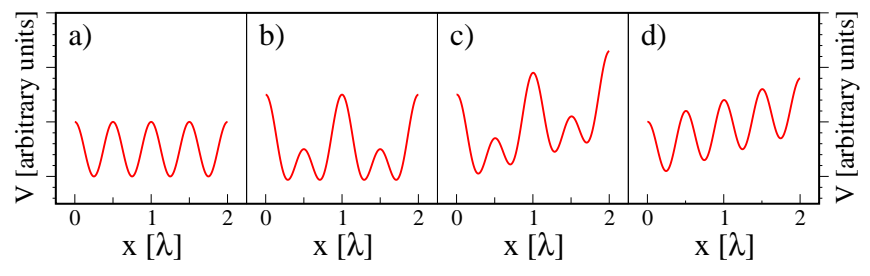

FIG. 1: Optical potentials generated by two counterpropagating laser beams with wave vector $k=2 \pi / \lambda$. a) $V=$ $V_{0} \sin (k x)^{2} ;$ b) $V=V_{0} \sin (k x)^{2}+V_{1} \sin (k x / 2)^{2}$; c) $V=$ $V_{0} \sin (k x)^{2}+V_{1} \sin (k x / 2)^{2}+V_{2} x ;$ d) $V=V_{0} \sin (k x)^{2}+V_{2} x$.

tools available for construction of $1 \mathrm{D}$ ladders and 2D square lattices with optical potentials $11,12,13,14,15]$. Counterpropagating laser beams along the $x_{i}(i=1,2,3)$ directions realize a periodic optical potential $V_{0}(\vec{x})=$ $\sum_{i} V_{0 i} \sin \left(k x_{i}\right)^{2}$ with wave vector $k=2 \pi / \lambda$, optical wave length $\lambda$, and lattice depths $V_{0 i}$ controlled by the laser intensities and atomic alternating current $(\mathrm{AC})$ polarizabilities. These potentials are additive, provided the lasers generating the lattice have slightly different optical frequencies, which are easily generated as sidebands from the original laser beam, so that the interference terms average out. Furthermore, interference of two lasers with angles $\pm \theta$ between their propagation directions give rise to potentials of the form $V_{1 i} \sin \left(k^{\prime} x_{i}+\Phi\right)^{2}$ with effective wave vector $k^{\prime}=k \cos \theta$ and displaced by a phase $\Phi$. This allows us to construct in particular potentials $V_{1}(\vec{x})=\sum_{i} V_{1 i} \sin \left(k x_{i} / 2\right)^{2}$. Adding these various potentials gives rise to superlattice structures. We can also add potentials in the form of linear ramps, $V_{2}(\vec{x})=\sum_{i} V_{2 i} x_{i}$ by sitting in the wing of a focused laser beam, as in far-off resonance laser traps. Fig. 1 illustrates lattice configurations which can be generated in this way by varying the intensity parameters $V_{0,1,2 i}$ which will be employed below for the construction of $d$-wave pairing.

The dynamics of cold atoms confined to these optical potentials is described by a Hubbard model 11, 12, 13, 14, 15.

$H=-\sum_{\langle i, j\rangle, \sigma} t_{i j}\left(c_{i \sigma}^{\dagger} c_{j \sigma}+\right.$ h.c. $)+U \sum_{i} n_{i \uparrow} n_{i \downarrow}+\sum_{i, \sigma} \mu_{i} n_{i \sigma}$,

where the $c_{i, \sigma}$ are fermionic annihilation operators with spin $\sigma$ and $n_{i, \sigma}=c_{i, \sigma}^{\dagger} c_{i, \sigma}$ is the particle number on site $i$. The $t_{i j}$ are spatially dependent hopping matrix elements connecting neighboring sites $i$ and $j$, and the $\mu_{i}$ are site offsets, as determined by the superlattice structure (see Fig. (1). The collisional repulsion $U$ between the atoms can be controlled by Feshbach resonances 19, 22. In writing the Hubbard model we have assumed that the optical potentials are spin independent, which is the usual case of Alkali atoms in their ground state 11].

Plaquette RVB States - The motion of the atoms can be confined to a $2 \mathrm{D}$ lattice by a strong transverse optical potential $V_{0 z}$. Employing superlattices we can gen-

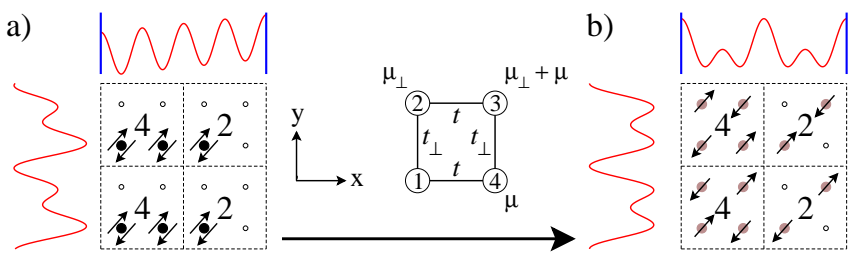

FIG. 2: Schematic illustration of the adiabatic protocol that generates RVB states on two decoupled plaquettes with 4 atoms (left) and 2 atoms (right). The protocol sequence is: 1) $t \rightarrow 1$; 2) $\mu \rightarrow 0$; 3) $\mu_{\perp} \rightarrow 0$. Optical potentials are sketched for $x$ and $y$-directions and the large (intermediate, small) circles indicate two (one, zero) atoms on a site.

erate double well potentials corresponding to decoupled plaquettes. Our first goal will be to study atomic dynamics on these uncoupled plaquettes, and in particular the generation of RVB (ground) states. The strategy is to (i) deform the optical lattice on each plaquette so that the corresponding ground state has a simple product form, $|\psi\rangle=\prod_{i \sigma} c_{i \sigma}^{\dagger}|0\rangle$, and (ii) adiabatically transform the lattice into an unperturbed plaquette, so that the final ground state is the desired RVB state. To prepare such a pure initial state we load atoms from a reservoir of a quantum degenerate Fermi gas via a coherent or dissipative Raman process into the optical lattice 23]. By choosing an appropriate pattern of site offsets in the lattice, we ensure only atoms are transferred which match the energy conservation condition. As shown in 23], this allows us to filter out from an initial finite entropy ensemble a pure spin and spatial pattern of atoms.

We consider preparation of one plaquette with 4 atoms (half-filling) and one plaquette with 2 atoms. The initial optical lattice for these two cases is described in Fig. 22), which amounts in the limit $t_{\perp} \ll \mu_{\perp}$ to a preparation of the states with $4(2)$ atoms in a product state of the form

$$
|4\rangle^{(0)}=c_{1 \uparrow}^{\dagger} c_{1 \downarrow}^{\dagger} c_{4 \uparrow}^{\dagger} c_{4 \downarrow}^{\dagger}|0\rangle, \quad|2\rangle^{(0)}=c_{1 \uparrow}^{\dagger} c_{1 \downarrow}^{\dagger}|0\rangle,
$$

where the indices 1 and 3 denote the wells along the $x$ direction as illustrated in Fig. 2]

An adiabatic protocol that allows to transform this initial product state to an RVB plaquette state is presented in Fig. 2 using the transformations of optical lattices illustrated in Fig. 1 Full plaquette symmetry is restored by coupling two wells first along the $x$-direction with their fully depleted counterparts along the $y$-direction. The initial optical lattice breaks reflection symmetry along the $x$-direction which upon coupling by increasing $t\left(V_{0 x} \rightarrow 0\right)$ enforces that the state $|2\rangle^{(0)}$ adiabatically connects only to the dimer state which is antisymmetric under spin exchange and symmetric under reflection. Eliminating the chemical potential shifts $\mu\left(V_{2 x} \rightarrow 0\right)$ and $\mu_{\perp}(\Phi \rightarrow 0)$ one can adiabatically prepare the ground states of a 4 -site plaquette state. For 4 atoms it is given 
a)

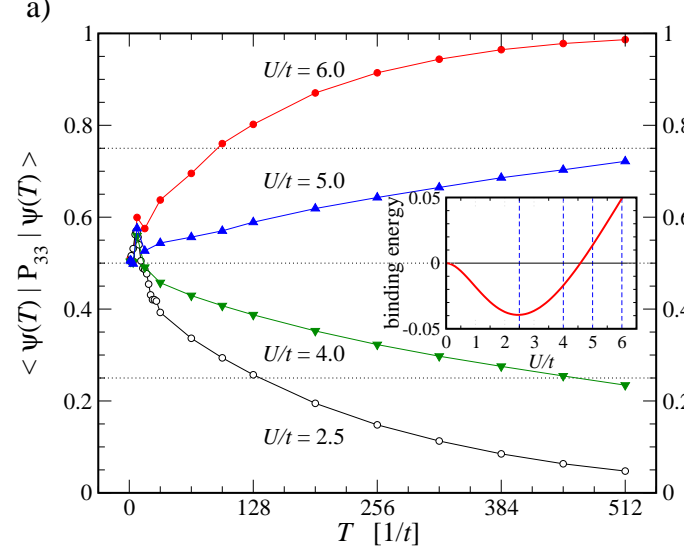

b)

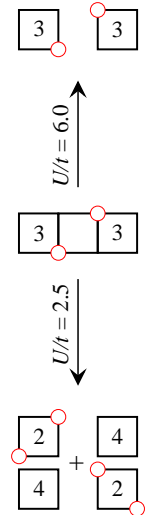

FIG. 3: Signature for $d$-wave RVB pairing when decoupling two plaquettes with 6 atoms for varying onsite repulsion $U / t$. b) For $U / t \lesssim 4.5$ pairs are formed with a small binding energy shown in the inset. In the final state after sufficiently slow decoupling the hole pair is located on one plaquette, while for large repulsion $U / t \gtrsim 4.5$ the unpaired holes separate into 3 atoms on each plaquette (right panel). a) Projection of the final state onto the subspace with 3 atoms on each plaquette.

by

$$
|4\rangle \approx \frac{1}{\sqrt{2}}\left(s_{1,2}^{\dagger} s_{3,4}^{\dagger}-s_{1,4}^{\dagger} s_{2,3}^{\dagger}\right)|0\rangle,
$$

where we have only written the dominant terms omitting states with local double occupancy, and $s_{i, j}^{\dagger}=$ $\left(c_{i \uparrow}^{\dagger} c_{j \downarrow}^{\dagger}-c_{i \downarrow}^{\dagger} c_{j \uparrow}^{\dagger}\right) / \sqrt{2}$ is a singlet state formed on sites $i$ and $j$. On the other plaquette the 2 atoms form a state

$|2\rangle \approx\left[\frac{1}{\sqrt{8}}\left(s_{1,2}^{\dagger}+s_{3,4}^{\dagger}+s_{1,4}^{\dagger}+s_{2,3}^{\dagger}\right)+\frac{1}{2}\left(s_{1,3}^{\dagger}+s_{2,4}^{\dagger}\right)\right]|0\rangle$.

While the state $|2\rangle$ has $s$-wave symmetry with respect

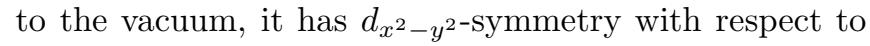
the ground state $|4\rangle$ at half-filling, as the bosonic $d$-wave hole pair operator $\Delta=\left(s_{1,2}+s_{3,4}-s_{1,4}-s_{2,3}\right) / 2$ has a non-vanishing contribution $\langle 2|\Delta| 4\rangle \neq 0$. The state $|2\rangle$ is therefore referred to as the $d$-wave RVB state (of the hole pair). The whole transformation is protected by a finite gap that sets the time scale for an adiabatic coupling with a fidelity larger than 0.99 to $T \approx 50 / t$.

Coupling of plaquettes - Coupling and decoupling these two plaquettes can provide a clear experimental signature for the pairing of atoms (or holes) in these RVB states, see Fig. 3. When coupling the plaquettes the holes move to the center of the 8 coupled sites as illustrated in Fig. 3b). Subsequently decoupling the two plaquettes, the various atomic occupation numbers on the two plaquettes are in direct competition. If a bound state of two atoms (holes) is disfavored by large onsite repulsion (inset of Fig. 3 $\mathrm{k}$ ), the atoms will maximize their respective kinetic energies as the plaquettes are decoupled and the holes separate. After adiabatic decoupling there are

two plaquettes with 3 atoms each as shown in Fig. 3 If a bound state is favorable the pair hops to either one of the plaquettes, leaving the system in a final state with an even number of atoms on both plaquettes. Pair binding is found for $U / t \lesssim 4.5$ with a maximum binding energy of $E_{b} / t \approx 0.04$ for $U / t \approx 2.5$ (see inset of Fig. [3] [ []]. The time scale for the adiabatic decoupling is dominated by the small binding energy scale and found to be $T \approx 500 / t$ or $T \approx 1 / 2$ seconds for ${ }^{40} \mathrm{~K}$ atoms which is well within the decoherence time of these systems [16]. Reversing the adiabatic protocol illustrated in Fig. 2 the plaquette states after decoupling are transformed into single-site states. For pair binding the system ends up with 3 doubly occupied sites, while for unbound pairs there will be two doubly occupied sites and two sites with one atom each. Experimentally the two scenarios can be distinguished by associating the atoms into molecules [15, 24] with the number of formed molecules having a ratio of $3 / 2$ respectively.

The detailed adiabatic protocol that couples two pla-

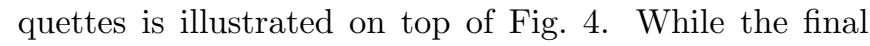
ground state of two plaquettes with 6 atoms is symmetric with respect to the exchange of the two plaquettes, coupling two plaquettes prepared in the states $|4\rangle$ and $|2\rangle$ will adiabatically connect the initial state $|4\rangle|2\rangle$ to both (anti)symmetric combinations $(|4\rangle|2\rangle \pm|2\rangle|4\rangle) / \sqrt{2}$. In order to prevent coupling to the antisymmetric state we need to explicitly break reflection symmetry along the $x$ direction. This can be achieved by ramping the chemical

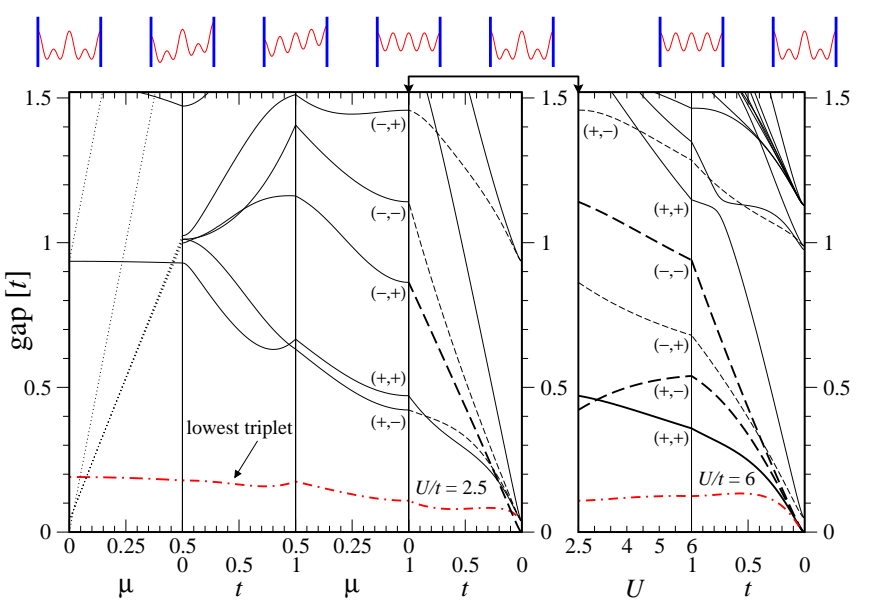

FIG. 4: Singlet gaps for the path that couples and decouples two plaquettes illustrated by the optical potentials on top. Two plaquettes with 4 and 2 atoms are coupled for $U / t=2.5$ with a chemical potential gradient $\mu$ being applied along the $x$-direction (first three panels). The decoupling of plaquettes is shown for bound (fourth panel) and unbound pairs (right panels). Dashed (dotted) lines indicate states to which a transition from the ground state is forbidden due to symmetry constraints (particle conservation on the individual plaquettes). The $x$ - and $y$-parities are given by "+" (even) and "-" (odd). 


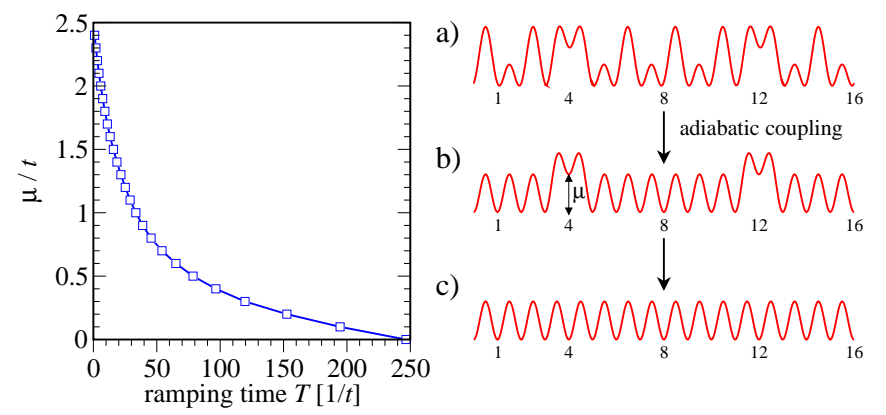

FIG. 5: Ramping times needed to achieve a fidelity larger than 0.999 for an adiabatically evolved wavefunction as the chemical potential shift $\mu$ at predefined lattice sites is reduced. The adiabatic protocol is performed in small sequences $\mu \rightarrow \mu-\delta \mu$ with $\delta \mu=0.1$. Doping $\delta=1 / 8$. On the right: a) decoupled plaquettes; b) comb-like structure; c) ladder geometry.

potential as illustrated in Fig. 15). If we subsequently increase the hopping between the two plaquettes $\left(V_{1 x} \rightarrow 0\right)$ and finally eliminate the shift in the chemical potential $\left(V_{2 x} \rightarrow 0\right)$, we can adiabatically connect the initial state to the symmetric ground state of two coupled plaquettes. The coupling transformation is protected by a considerable gap as shown in Fig. (4. The gaps for the subsequent decoupling process which keeps full exchange symmetry between the two plaquettes reveal the small pair binding energy in the vicinity of small interplaquette hopping $t$ as shown in Fig. 4 for $U / t=2.5$ (pair binding) and $U / t=6.0$ (no binding). The low-energy dynamics shown in Fig. [ $\mathrm{U}$ can be probed by measuring the structure factor in light-scattering spectroscopy [25].

Doped d-wave RVB ladders - Finally, we discuss halffilled ladder systems by coupling multiple plaquettes and the preparation of a hole doped $d$-wave RVB state. $d$ wave hole pairing in ladders is strongest for $U / t \approx 2.5$; pairs are localized and pinned with a period $1 / \delta$ for open boundary conditions and doping $\delta$. To enable a fast adiabatic coupling process we prepare an initial optical lattice that already places hole pairs close to their final location in the doped ladder by mimicking the $1 / \delta$ periodicity as shown in Fig. 5]). Using a pattern loading technique 23] we can load a gas of fermionic atoms into this optical lattice so that the shifted sites in this comb-like structure remain depleted while all other rungs in the ladder system are half-filled. When adiabatically coupling this initial state to the final RVB state of the doped half-filled ladder by reducing the chemical potential shifts $\mu$, the time scale is dominated by the time needed to establish phase coherence between previously unconnected ladder parts.

In our numerical simulations, we considered a ladder with 32 rungs filled with 56 particles, i.e. doping $\delta=1 / 8$. In the initial configuration 28 rungs are half-filled and the rungs 4, 12, 20, 28 depleted, see Fig. 5b). Using adaptive time-dependent density matrix renormalization group (DMRG) algorithms [26], we have calculated the time scales for ramping down $\mu$ at the specified sites. We find that with an overall ramping time $T \approx 250 / t$, following the protocol shown in Fig. (5), the fidelity is larger than 0.9 . To improve this bound to $\approx 0.99$, we estimate $T \sim 450 / t$.

Phase coherence in the ladder RVB state can be experimentally probed via molecule formation where the molecules are generated through a laser-induced Raman transition that couples to a molecular state $m$ with

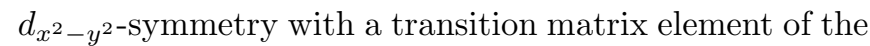
form $m^{\dagger} \Delta$ [15]. If the ladder exhibits quasi-long range order the generated molecules will be phase coherent forming a quasi-condensate. A truly long-range superfluid ground state of $d$-wave RVB pairs can be stabilized in experiment by weak coupling of ladders. Increasing the inter-ladder coupling in the experimental setup will answer one of the foremost open questions of solid state physics: is the $d$-wave RVB state on ladders adiabatically connected to the ground state of the Hubbard model on the uniform square lattice, and hence the RVB theory of high temperature superconductivity confirmed - or is there a quantum phase transition to a new phase?

ST acknowledges support by the Swiss National Science Foundation. Research at the University of Innsbruck is supported by the Austrian Science Foundation and EU projects. We thank the Aspen Center for Physics and the Kavli Institute for Theoretical Physics in Santa Barbara, where this work was initiated. Some of the simulations were based on the ALPS libraries [27].

[1] P.W. Anderson, Mater. Res. Bull. 8, 153 (1973); P. Fazekas and P.W. Anderson, Phil. Mag. 30, 432 (1974).

[2] J.G. Bednorz and K.A. Mueller, Z. Phys. B 64, 189 (1986).

[3] P.W. Anderson, Science 235, 1196 (1987).

[4] I. Affleck et al., Phys. Rev. B 38, 745 (1988); C. Gros, Phys. Rev. B 38931 (1988); G. Kotliar and J. Liu, Phys. Rev. B 38, 5142 (1988).

[5] C.C. Tsuei and J.R. Kirtley, Rev. Mod. Phys. 72, 969 (2000).

[6] P.W. Anderson et al., J. Phys.: Condens. Matter 16, R755 (2004).

[7] R.M. Fye et al., Phys. Rev. B 46, 8667 (1992).

[8] E. Altman and A. Auerbach, Phys. Rev. B 65, 104508 (2002).

[9] H. Tsunetsugu et al., Phys. Rev. B 49, 16078 (1994); M. Troyer et al., Phys. Rev. B 53, 251 (1996); R.M. Noack et al., Phys. Rev. Lett. 73, 882 (1994).

[10] H. Tsunetsugu et al., Phys. Rev. B 51, 16456 (1995); E. Arrigoni et al., Phys. Rev. B 69, 214519 (2004).

[11] D. Jaksch et al., Phys. Rev. Lett. 81, 3108 (1998); For a review see: D. Jaksch and P. Zoller, Annals of Physics 315, 52 (2005).

[12] W. Hofstetter et al., Phys. Rev. Lett. 89, 220407 (2002).

[13] L. Santos et al., Phys. Rev. Lett. 93, 030601 (2004). 
[14] A.S. Sorensen et al., Phys. Rev. Lett. 94, 86803 (2005).

[15] H.P. Büchler et al., Phys. Rev. Lett. 95, 040402 (2005).

[16] M. Köhl et al., Phys. Rev. Lett. 94, 080403 (2005).

[17] G. Roati et al., Phys. Rev. Lett. 92, 230402 (2004)

[18] B. Paredes et al., Nature 429, 277 (2004).

[19] M. Greiner, C.A. Regal, and D.S. Jin, Nature 426, 537 (2003); J. Cubizolles et al., Phys. Rev. Lett. 91, 240401 (2003); C. Chin et al., Science 305, 1128 (2004); M. W. Zwierlein et al., Nature 435, 1047 (2005).

[20] E. Farhi et al., Science 292, 472(2001).

[21] M. Troyer and U.-J. Wiese, Phys. Rev. Lett. 94, 170201 (2005).
[22] M.W.J Romans et al., Phys. Rev. Lett. 93, 020405 (2004).

[23] P. Rabl et al., Phys. Rev. Lett. 91, 110403 (2003); A. Griessner et al., Phys. Rev. A 72, 032332 (2005).

[24] T. Rom et al., Phys. Rev. Lett. 93, 073002 (2004).

[25] S. Trebst et al., in preparation

[26] A.J. Daley et al., J. Stat. Mech. P04005 (2004); S.R. White and A.E. Feiguin, Phys. Rev. Lett. 93, 076401 (2004); A.E. Feiguin and S.R. White, cond-mat/0502475

[27] F. Alet et al., J. Phys. Soc. Jpn. Suppl. 74, 30 (2005). 